\title{
Microestrutura e Propriedades de Desgaste do Aço Bainítico de Resfriamento Contínuo DIN 18MnCrSiMo6-4 Nitretado a Plasma em Diferentes Temperaturas
}

\section{Microstructure and Wear Properties of DIN 18MnCrSiMo6-4 Continuous Cooling Bainitic Steel Plasma Nitrided at Different Temperatures}

Rafael Luciano Dalcin ${ }^{1}$, Leonardo Fonseca Oliveira ${ }^{1}$, Alexandre da Silva Rocha ${ }^{1}$, Célia de Fraga Malfatti ${ }^{2}$, Gelsa Edith Navarro Hidalgo ${ }^{3}$, Jérémy Epp ${ }^{4}$, Hans-Werner Zoch ${ }^{4}$

\footnotetext{
${ }^{1}$ Laboratório de Transformação Mecânica - LdTM - PPGE3M/UFRGS, Av. Bento Gonçalves, 9500, CEP: 91501-970, Porto Alegre, RS, Brasil.

${ }^{2}$ Laboratório de Pesquisa em Corrosão - LAPEC - PPGE3M/UFRGS, Av. Bento Gonçalves, 9500, CEP: 91501-970, Porto Alegre, RS, Brasil.

${ }^{3}$ Laboratório de Processamento Mineral - LAPROM - PPGE3M/UFRGS, Av. Bento Gonçalves, 9500, CEP: 91501-970, Porto Alegre, RS, Brasil.

${ }^{4}$ Institut für Werkstofforientierte Technologien - LEIBNIZ - IWT, Badgasteiner Strasse 3, 28359, Bremen, Bremen, Alemanha.

e-mail: rafael.dalcin@ufrgs.br, leonardo.fonseca@ufrgs.br, celia.malfatti@ufrgs.br, gelsaedith@ufrgs.br, alexandre.rocha@ufrgs.br, epp@iwt-bremen.de, zoch@iwt-bremen.de
}

\section{RESUMO}

Os novos aços bainíticos de resfriamento contínuo (CCBS) são uma excelente alternativa quando se requer elevada dureza, tenacidade e resistência à fadiga. Contudo, estima-se que a taxa de desgaste dos CCBS seja insuficiente para aplicação em componentes com alta solicitação mecânica, sendo necessário aprimorar suas propriedades de superfície. O objetivo deste estudo é o de analisar os efeitos da nitretação a plasma sobre à microestrutura e propriedades de desgaste do aço bainítico de resfriamento contínuo DIN18MnCrSiMo6-4. Portanto, tratamentos com duração de 6 horas foram conduzidos com pressão de 3 mbar, mistura gasosa composta por $76 \% \mathrm{~N}_{2}+24 \% \mathrm{H}_{2}$, e temperaturas de $400,450,500$ e $550{ }^{\circ} \mathrm{C}$, respectivamente. As amostras foram analisadas através de microscopia eletrônica de varredura, difração de raios-X, microdureza e ensaios de desgaste por deslizamento recíproco. Nas condições investigadas, foi possível obter uma camada de compostos e atingir um aumento de até $393 \%$ na dureza superficial, entretanto, o uso de temperaturas mais elevadas durante os tratamentos favoreceu a formação de camadas mais profundas. Em todas as amostras nitretadas, constatou-se a formação predominante da fase de nitretos $\varepsilon-\mathrm{Fe}_{2-3} \mathrm{~N}$ e, em menor quantidade, da fase de nitretos $\gamma^{\prime}-\mathrm{Fe}_{4} \mathrm{~N}$. Nos ensaios de deslizamento recíproco houve diminuição do desgaste das amostras nitretadas na temperatura de $550^{\circ} \mathrm{C}$.

Palavras-chave: Aços Bainíticos de Resfriamento Contínuo, Nitretação a Plasma, Microestrutura e Propriedades de Desgaste.

\footnotetext{
ABSTRACT

The new continuous cooling bainitic steels (CCBS) are an excellent alternative when high hardness, toughness and fatigue resistance are required. However, it is estimated that the wear rate of the CCBS is insufficient for application in highly mechanical loaded components, being necessary to improve the surface properties. The objective of this study is to analyze the effects of plasma nitriding on the microstructure and wear properties of DIN18MnCrSiMo6-4 continuous cooling bainitic steel. Therefore, treatments lasting 6 hours were conducted with a pressure of 3 mbar, a gas mixture composed of $76 \% \mathrm{~N}_{2}+24 \% \mathrm{H}_{2}$, and a temperature of $400,450,500$ and $550^{\circ} \mathrm{C}$, respectively. The samples were analyzed by scanning electron microscopy, $\mathrm{X}$ -
} 
ray diffraction, microhardness and reciprocal sliding wear tests. Under the conditions investigated, it was possible to obtain a compound layer and to achieve an increase of up to $393 \%$ in surface hardness, however, the use of higher processing temperatures favored the extension of the depth layer. In all the nitrided samples, the major presence of $\varepsilon-\mathrm{Fe}_{2-3} \mathrm{~N}$ nitrides phase was observed, and the $\gamma^{\prime}-\mathrm{Fe}_{4} \mathrm{~N}$ phase content was smaller. In the reciprocal sliding tests, there was a decrease in the wear of nitrided samples at temperature of $550{ }^{\circ} \mathrm{C}$.

Keywords: Continuous Cooling Bainitic Steel, Plasma Nitriding, Microstructure and Wear Properties.

\section{INTRODUÇÃO}

Estima-se um aumento de 7\% em 2009 para até 36\% em 2020 na proporção dos aços de alta resistência mecânica utilizados em componentes automotivos [1]. Nesse sentido, a indústria siderúrgica vem desenvolvendo novos aços com propriedades metalúrgicas e métodos de processamento exclusivos, que permitem que a indústria automotiva atenda aos requisitos de segurança, eficiência, emissões, fabricação, durabilidade e qualidade a um custo relativamente baixo [2-8]. Simultaneamente, muitos grupos de pesquisa estão realizando estudos sobre esses novos tipos de aço para entender melhor suas aplicações [9] e encurtar a rota de processamento [10-13]. Uma alternativa que tem sido tentada é o desenvolvimento de novas microestruturas [1425].

Técnicas de produção de aços envolvendo micro adição de elementos de liga e estritos controles do aquecimento, da laminação e do resfriamento produzem microestruturas capazes de desenvolver ótimas propriedades mecânicas, que dentro de certos limites de resistência e espessura podem ser alcançadas sem a necessidade de tratamento térmico após a laminação. De forma geral, esse procedimento é denominado processamento termomecânico e através dele são obtidas diversas categorias de aços [26,27]. O processamento termomecânico é um processo metalúrgico que integra as operações de conformação e tratamento térmico em uma única etapa para controlar a microestrutura dos materiais, e, consequentemente, melhorar suas propriedades mecânicas durante o processo de conformação a quente [8,28].

A nova geração de aços bainíticos formados durante o resfriamento contínuo permite a formação de diferentes morfologias bainíticas (bainita granular, bainita superior tipo ripa, bainita inferior, etc.) e pode conter outras fases como austenita retida e ferrita [29-31]. Ao contrário dos aços temperados e revenidos, que necessitam de posterior tratamento térmico para obter propriedades adequadas para suas aplicações, os aços bainíticos resfriados continuamente já apresentam elevados níveis de resistência e tenacidade após laminação a quente ou forjamento, sem necessidade de tratamento térmico posterior, apresentando potencial para encurtar o ciclo de fabricação, reduzindo custos [30,32,33].

A possibilidade de associar tratamentos termoquímicos com a boa qualidade estrutural dos aços bainíticos tem despertado interesse de vários pesquisadores [34-36]. De acordo com Lembke et al. [34], a dureza superficial dos aços bainíticos após a nitretação pode ser comparada com a dos aços temperados e revenidos, tornando a técnica atrativa para muitos componentes automotivos sujeitos a altas cargas dinâmicas, desgaste e corrosão [37-41]. A nitretação a plasma, por permitir trabalhar em temperaturas mais baixas que os processos termoquímicos tradicionais, é uma opção eficiente para aumentar a dureza superficial de componentes mecânicos, sem prejudicar as propriedades do núcleo do material [42-46]. Portanto, o propósito desta investigação é verificar a influência da nitretação a plasma sobre a microestrutura e propriedades de desgaste do aço bainítico de resfriamento contínuo DIN 18MnCrSiMo6-4.

\section{MATERIAIS E MÉTODOS}

Amostras cilíndricas do aço bainítico de resfriamento contínuo DIN 18MnCrSiMo6-4 com diâmetro 43,0 mm e 10,0 mm de espessura foram utilizadas para a realização dos tratamentos de nitretação a plasma. A microestrutura do aço DIN 18MnCrSiMo6-4 é composta basicamente por ferrita pró-eutetóide, bainita granular e constituintes martensita/austenita (M/A) $[20,29,35]$. O percentual de elementos químicos e propriedades mecânicas do aço DIN 18MnCrSiMo6-4 são apresentados na Tabela 1.

Tabela 1: Composição química e propriedades mecânicas do aço DIN 18MnCrSiMo6-4.

\begin{tabular}{|c|c|c|c|c|c|c|c|c|}
\hline \multicolumn{9}{|c|}{ ELEMENTOS QUÍMICOS (\% EM MASSA) } \\
\hline C & Si & Mn & $\mathbf{P}$ & $\mathbf{S}$ & $\mathrm{Cr}$ & Mo & $\mathrm{Ni}$ & Al \\
\hline 0,19 & 1,16 & 1,35 & 0,01 & 0,015 & 1,14 & 0,26 & 0,06 & 0,01 \\
\hline Co & $\mathrm{Cu}$ & $\mathrm{Nb}$ & Mg & $\mathbf{v}$ & $\mathrm{Ti}$ & w & Sn & B \\
\hline$<0,01$ & 0,09 & $<0,001$ & - & $<0,001$ & 0,002 & $<0,01$ & 0,01 & 0,001 \\
\hline
\end{tabular}




\begin{tabular}{llll}
\hline PROPRIEDADES MECÂNICAS (VALORES MÉDIOS) & & \\
\hline Tensão de Escoamento & Limite de Resistência à Tração & Alongamento & Dureza \\
\hline $825 \mathrm{MPa}$ & $1017 \mathrm{MPa}$ & $17 \%$ & $330 \mathrm{HV}$ \\
\hline
\end{tabular}

Para garantir condições de pré-tratamento semelhantes entre os substratos, as superfícies planas das amostras foram preparadas com lixamento gradual, utilizando lixas d'água de granulometria variável de 80 a 1200 mesh, alternando o sentido em $90^{\circ}$ em cada etapa do lixamento [47]. Posteriormente, foi realizado polimento com pasta de diamante de $3 \mu \mathrm{m}$. Antes da realização do tratamento de nitretação a plasma, as amostras utilizadas foram limpas com algodão embebido em álcool etílico e acetona. Os tratamentos de nitretação a plasma foram conduzidos em um forno reator (tipo sino), com duração de 6 horas na temperatura de 550, 500,450 e $400{ }^{\circ} \mathrm{C}$, respectivamente. As amostras foram nitretadas com pressão de 3 mbar e mistura gasosa constituída por $76 \%$ de $\mathrm{N}_{2}+24 \% \mathrm{H}_{2}$, em volume. Após a nitretação a plasma, as amostras foram lentamente resfriadas no vácuo até a temperatura ambiente. Várias técnicas foram usadas para caracterizar a estrutura e as propriedades mecânicas das amostras nitretadas e não nitretadas do aço DIN 18MnCrSiMo6-4. As análises incluíram microscopia ótica (MO), microscopia eletrônica de varredura (MEV), análise de fases por difração de raios- $\mathrm{X}$, ensaios de microdureza e ensaios de desgaste.

As análises de estrutura e composição incluíram análise de difração de raios-X para identificação de camadas nitretadas e MEV para a determinação da estrutura superficial e transversal das amostras. Os ensaios de difração de raios-X foram conduzidos em um difratômetro GE Seifiert Charon XRD M-Research Edition equipado com tubo de raios-X de radiação cromo, com comprimento de onda de 2,2897 $\AA$. Os ângulos $2 \theta$ analisados compreendem um intervalo de $50^{\circ}$ a $166^{\circ}$, com espaçamento de $0,01^{\circ}$ e tempo de coleta de $200 \mathrm{~s}$ em cada passo. As fases presentes no aço e nas camadas nitretadas foram determinadas por análise comparativa entre padrões contidos nos arquivos de informações cristalográficas (CIFs) oriundos do Crystallography Open Database [48] e do Inorganic Crystal Structures Database [49] por intermédio do software PROFEX$B G M N$ [50]. As análises da microestrutura e das regiões afetadas pela nitretação foram efetuadas no equipamento MEV TESCAN VEGA3.

As análises das propriedades mecânicas incluem ensaios de microdureza e ensaio de desgaste por deslizamento recíproco. As medidas de microdureza Vickers foram realizadas com o microdurômetro INSIZE $I S H-T D V$ 1000, na seção transversal das amostras nitretadas para verificar o gradiente de dureza formado na zona superficial afetada pela nitretação. Cinco perfis de microdureza foram construídos usando um indentador Vickers sob carga de 100 gf durante 10 segundos [51]. A determinação da profundidade de camada nitretada foi feita com base nos perfis de microdureza, e seguindo recomendações estabelecidas na norma DIN 50 190 [52]. Os ensaios de desgaste foram realizados em temperatura ambiente com um tribômetro CETR-UMT de deslizamento linear recíproco ball-on-flat. Quatro trilhas de desgaste com comprimento de $4 \mathrm{~mm}$ foram produzidas sobre a superfície de cada amostra. Durante os ensaios, esferas de alumina $\left(\mathrm{Al}_{2} \mathrm{O}_{3}\right)$ com diâmetro $4,76 \mathrm{~mm}$ são pressionadas com carga de $6 \mathrm{~N}$ contra a superfície das amostras. O deslizamento prossegue sobre a trilha de $4 \mathrm{~mm}$ durante 30 minutos numa frequência de $4 \mathrm{~Hz}$. Após a realização dos ensaios de desgaste, as superfícies das trilhas de desgaste foram analisadas em um microscópio óptico OLYMPUS GX-51 e em um perfilômetro interferômetro tridimensional Bruker Contour Elite para a quantificação do desgaste ocorrido nas amostras.

\section{RESULTADOS}

\subsection{Caracterização da Camada Nitretada}

A estrutura da superfície das amostras nitretadas são mostradas na Figura 1. No substrato mostrado na Figura 1 aparecem como fases claras a presença de constituintes martensita/austenita (M/A). A matriz cinza escura é composta por ferrita e bainita. Inclusões muito finas de carbonetos de cromo podem ser encontradas dentro da matriz bainítica [35,53]. Na zona de difusão, logo abaixo da camada de compostos, é revelada uma clara descarbonetação [54,55].

A Figura 2 apresenta os resultados das medições de profundidade total de camada (camada de compostos + zona de difusão), construídos a partir dos perfis de microdureza Vickers e da espessura da camada de compostos por exame metalográfico ao microscópio ótico. Todas as amostras nitretadas apresentaram uma camada de compostos depositados na superfície. Isto foi comprovado nos difratogramas de raios-X, Figura 3.

A Figura 3 apresenta o difratograma das amostras nitretadas a plasma e de uma amostra não nitretada utilizada como referência. A amostra do aço não nitretado é caracterizada basicamente pela presença da fase 
de ferro $\alpha$-Fe nas posições: $68,8^{\circ} ; 106,1^{\circ}$ e $156,4^{\circ}$ e de austenita retida $\gamma$-Fe nas posições: $66,9^{\circ} ; 79,2^{\circ}$ e $128,4^{\circ}$. Todas as amostras nitretadas apresentaram picos de difração em mais de uma posição $2 \theta$, indicando a formação da camada de compostos nestes tratamentos. No difratograma apresentado, verifica-se a presença majoritária de nitretos $\varepsilon-\mathrm{Fe}_{2-3}(\mathrm{C}) \mathrm{N}$ nas posições: $58,2^{\circ} ; 62,9^{\circ} ; 67,0^{\circ} ; 91,0^{\circ} ; 114,8^{\circ} ; 134,3^{\circ} ; 153,2^{\circ}$ e $164,5^{\circ}$, e também indícios de nitretos $\gamma^{\prime}-\mathrm{Fe}_{4} \mathrm{~N}$ nas posições: $63,1^{\circ} ; 74,3^{\circ}$ e $117,4^{\circ}$.

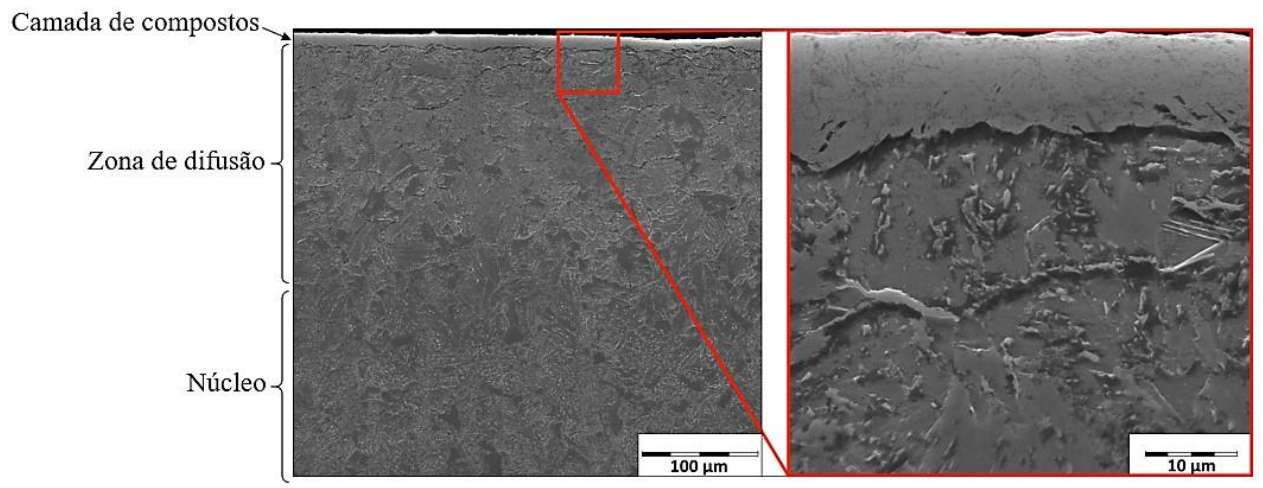

$\left(550{ }^{\circ} \mathrm{C}\right)$

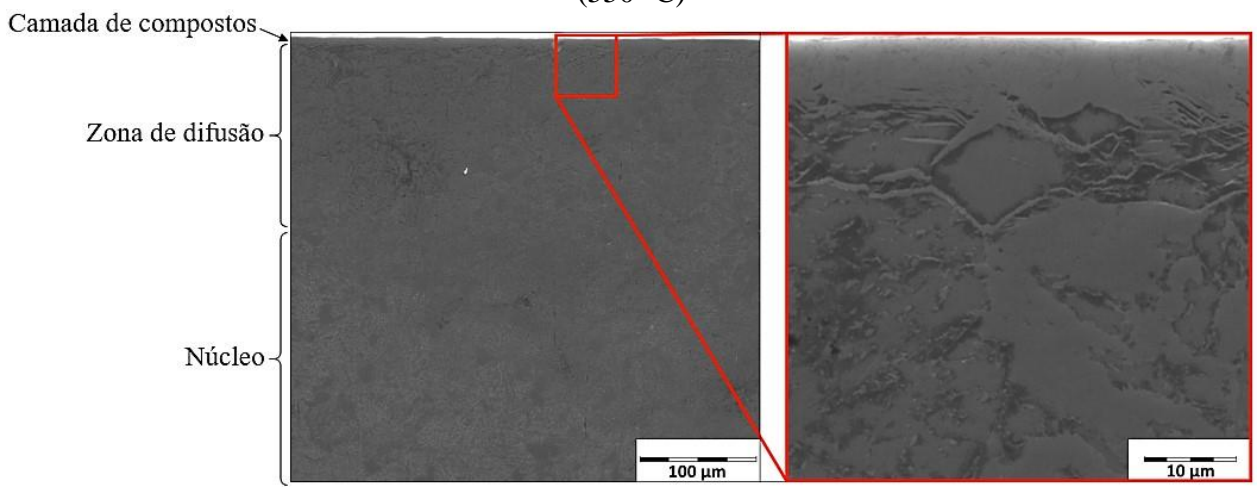

$\left(500{ }^{\circ} \mathrm{C}\right)$

Camada de compostos

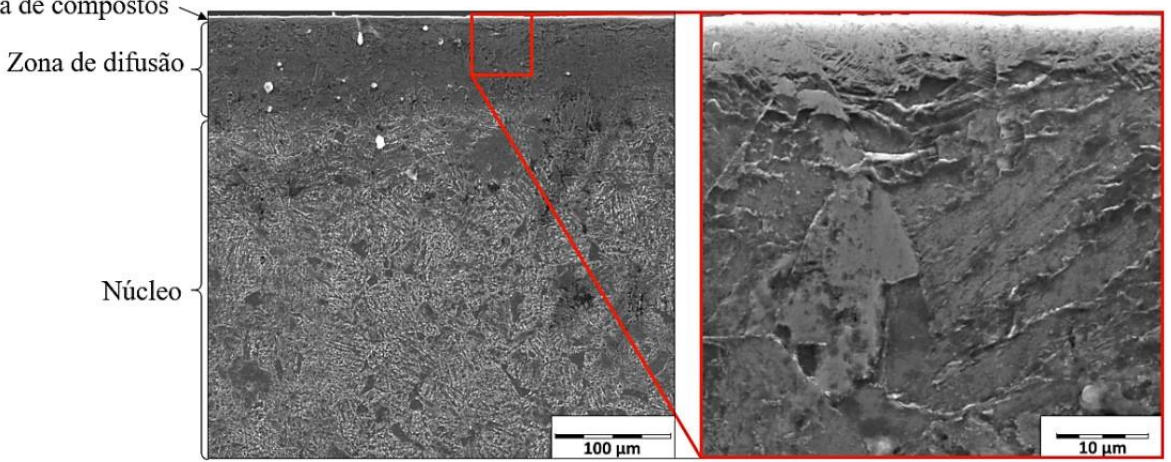

$\left(450{ }^{\circ} \mathrm{C}\right)$

Camada de compostos

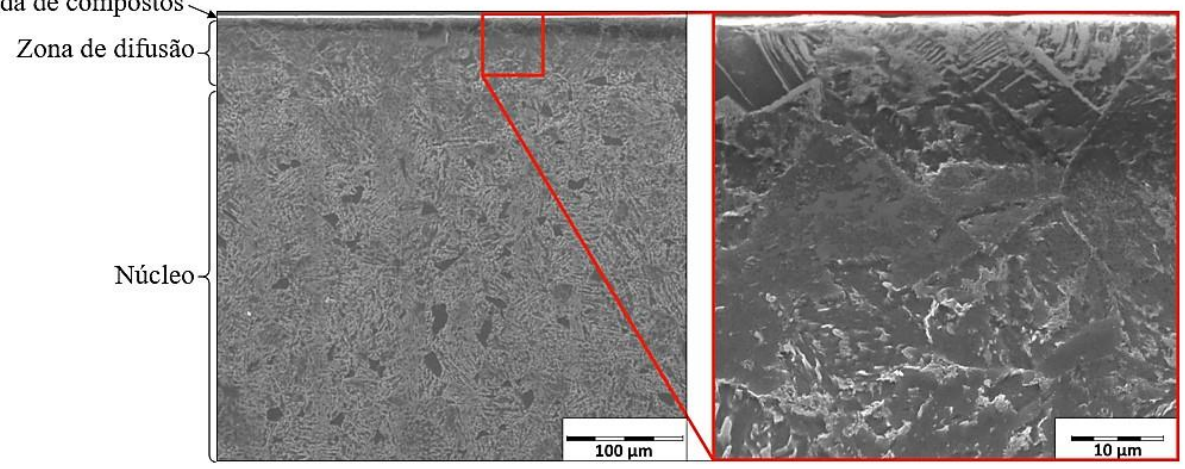

$\left(400{ }^{\circ} \mathrm{C}\right)$

Figura 1: Micrografias da superfície das amostras nitretadas a plasma. 


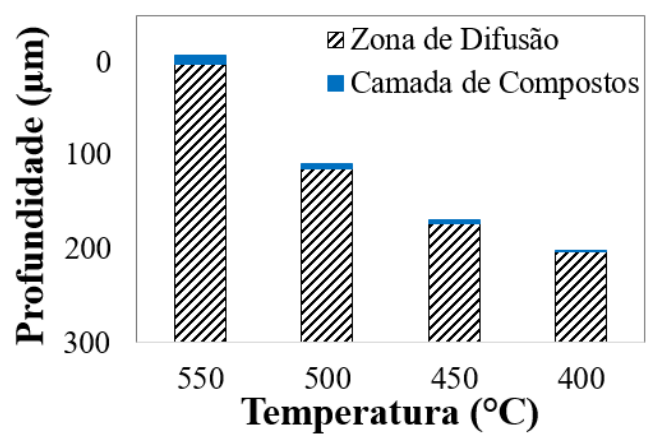

Figura 2: Profundidade de camada das amostras nitretadas a plasma.

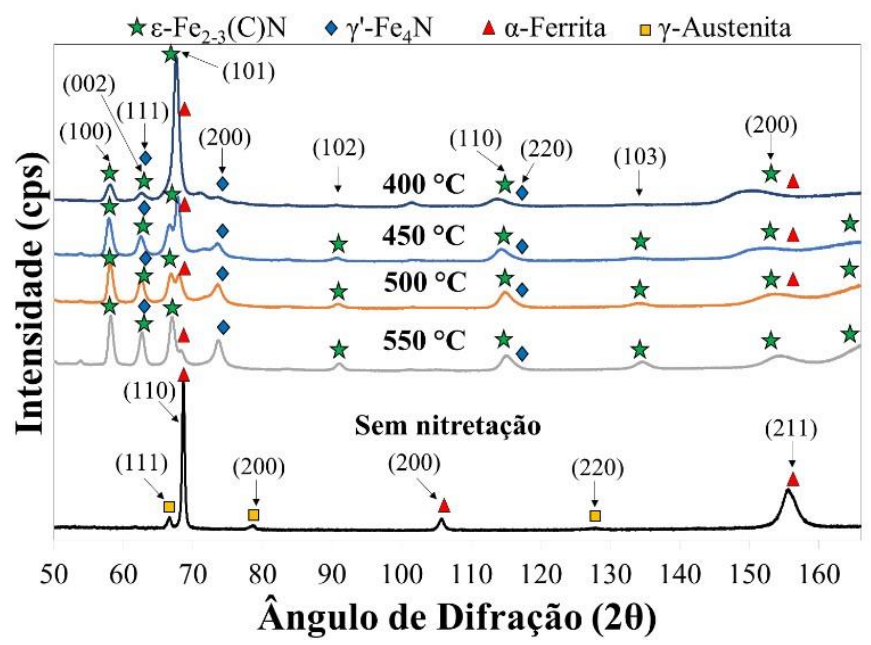

Figura 3: Difratogramas de raio- $X$ das amostras nitretadas e da amostra não nitretada.

\subsection{Propriedades Mecânicas e de Desgaste}

Os perfis de microdureza das amostras nitretadas a plasma são apresentados na Figura 4. A dureza diminui gradativamente da superfície para o núcleo, uma vez que a concentração de nitrogênio diminui em direção ao núcleo [56]. A dureza superficial máxima é obtida na amostra nitretada na temperatura de $500{ }^{\circ} \mathrm{C}$. Neste caso, a dureza aumentou 393\%, passando do valor original de 330 para $1300 \mathrm{HV}$. As durezas superficiais mais baixas (1088 e $1050 \mathrm{HV}$ ) foram encontradas nas amostras nitretadas na temperatura de 550 e $400{ }^{\circ} \mathrm{C}$, respectivamente.

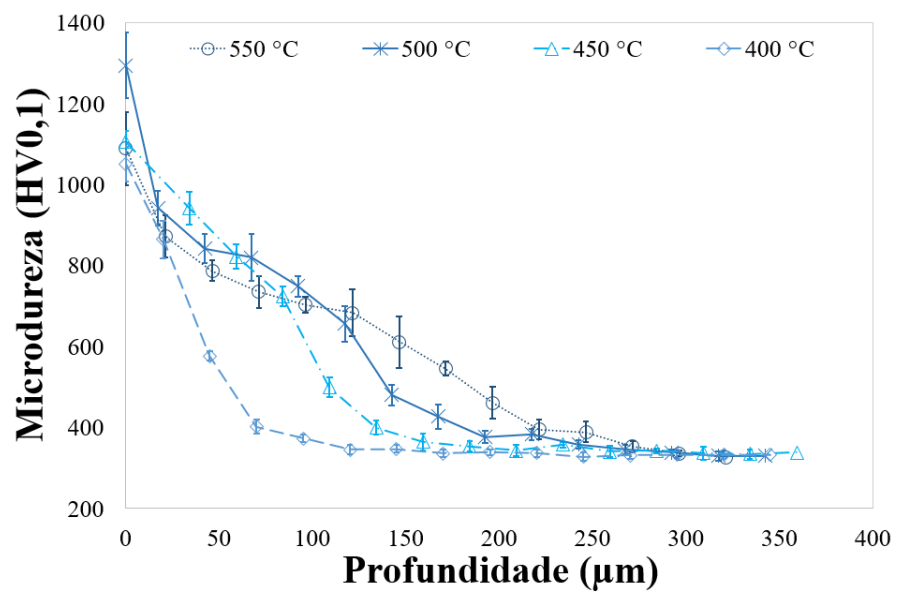

Figura 4: Variação de dureza após a nitretação a plasma.

O coeficiente de atrito medido das amostras nitretadas e da amostra não nitretada durante os ensaios 
de desgaste por deslizamento recíproco revelou um comportamento diferente entre as superfícies investigadas, Figura 5. É possível observar que as amostras nitretadas apresentam reduzido coeficiente de atrito em comparação com a amostra não nitretada durante os estágios iniciais do ensaio de desgaste ball-on-flat. Isto pode ser atribuído ao aumento de dureza causado pela nitretação, refletindo em menores deformações superficiais durante o deslizamento contra a esfera de alumina, havendo menor área de contato entre os corpos e, portanto, menor resistência ao deslizamento $[55,57,58]$. A medida que a distância do deslizamento aumenta, observase um aumento gradual do coeficiente de atrito ao passo que as amostras vão sendo desgastadas. Por outro lado, o aumento repentino do coeficiente de atrito observado nas amostras nitretadas na temperatura de 400 , 450 e $500{ }^{\circ} \mathrm{C}$, pode estar relacionado com topografia da superfície e na maneira como ocorreu o desgaste em cada amostra $[59,60]$. A amostra nitretada na temperatura de $550{ }^{\circ} \mathrm{C}$ apresentou um aumento gradual do coeficiente de atrito que culmina em um regime estacionário sem o aumento repentino observado nas outras amostras nitretadas em temperaturas mais baixas. Na amostra nitretada em $550^{\circ} \mathrm{C}$ houve também a formação de um filme superficial, responsável pela forte redução do coeficiente de atrito. Esse filme que pode ser óxidos e/ou compostos orgânicos a base de $\mathrm{O}, \mathrm{N}$ e C resultaram em efeito lubrificante, reduzindo o atrito.

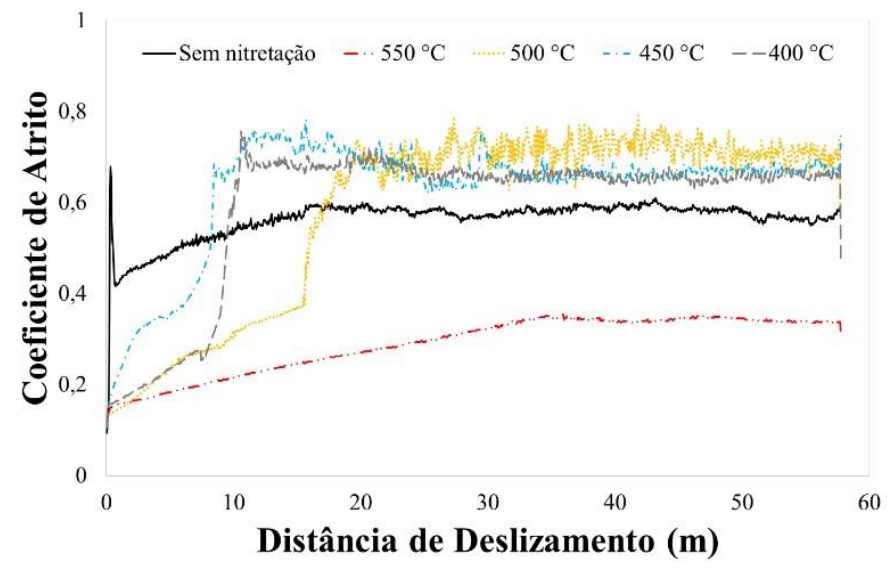

Figura 5: Coeficiente de atrito de amostras nitretadas e da amostra não nitretada.

A Figura 6 exibe o perfil transversal e a estrutura das trilhas de desgaste de amostras não nitretadas e de amostras nitretadas a plasma nas temperaturas de $550,500,450$ e $400{ }^{\circ} \mathrm{C}$. No aço sem nitretação, a característica marcante é a presença de marcas de desgaste na forma de riscos e ranhuras, o que revela que o desgaste é do tipo abrasivo [61]. De modo geral, nas amostras nitretadas a plasma a presença destas marcas é muito menor, já que o aumento da dureza inibe a deformação plástica e o arrancamento de material da superfície. No entanto, a piora na resistência ao desgaste das amostras nitretadas em 500,450 e $400{ }^{\circ} \mathrm{C}$ pode ser devido a formação de precipitados em contorno de grão [62], ou seja, uma fragilização na zona de difusão.

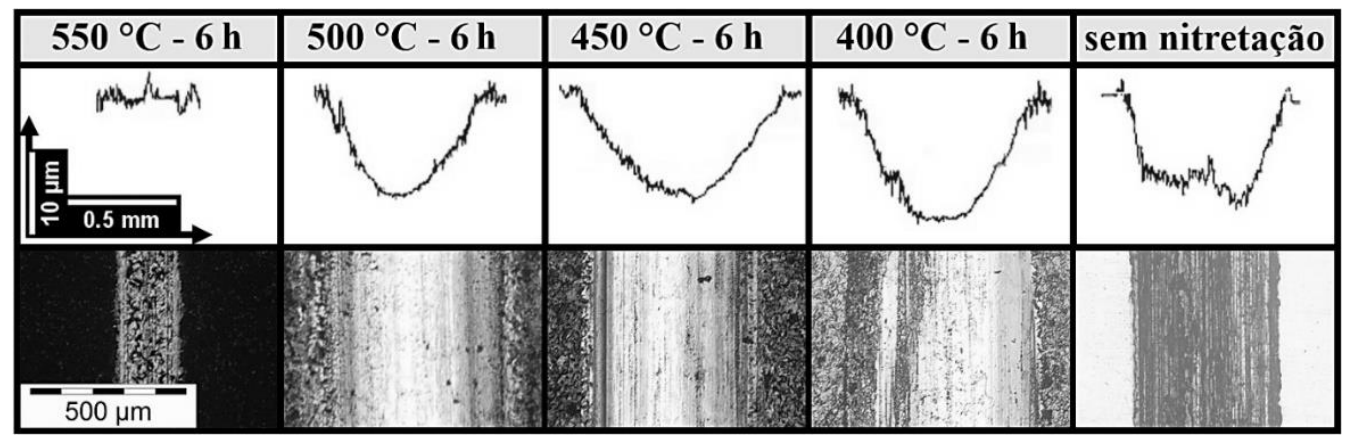

Figura 6: Perfil transversal e estrutura das trilhas de desgaste de amostras nitretadas e da amostra não nitretada.

\section{DISCUSSÃO}

De acordo com Diehl et al. [55], Tier et al. [63] e Kwietniewski et al. [64] na Figura 1 existe o efeito de desestabilização dos carbonetos formados pelos elementos de liga do aço, em especial o carboneto de cromo, durante a nitretação. Os elementos de liga que antes formavam carbonetos formam novos compostos com o 
nitrogênio (nitretos), deixando o carbono livre para se difundir em duas direções dentro da peça: em direção à sua superfície e em direção ao seu núcleo. O carbono que se difunde para o núcleo, região livre de tensões residuais, devido à formação de novos nitretos [54] forma uma zona rica em carbono abaixo da camada nitretada, que apresenta um teor maior que aquele anterior ao tratamento [55].

Como a inserção do nitrogênio na superfície é um processo difusional [65], o uso de temperatura mais elevada favoreceu a ampliação da profundidade de camada [56], Figura 2. Enquanto que no tratamento a $550{ }^{\circ} \mathrm{C}$ a camada de compostos alcançou aproximadamente $9,6 \mu \mathrm{m}$, nos tratamentos a 500,450 e $400{ }^{\circ} \mathrm{C}$ as camadas alcançaram cerca de 6,$7 ; 3,9$ e $1,5 \mu \mathrm{m}$. A espessura da zona de difusão das amostras nitretadas em $550,500,450$ e $400{ }^{\circ} \mathrm{C}$ é 299,$0 ; 185,7 ; 127,2$ e $97,0 \mu \mathrm{m}$, respectivamente.

Não é possível distinguir com clareza se a fase $\alpha$-Fe é ferrita, bainita ou martensita na Figura 3. O deslocamento do pico para a esquerda indica um aumento das tensões residuais de segunda e primeira ordem [55]. O alargamento do pico, devido a maior densidade de discordâncias, indica a presença de bainita/martensita [66]. Os picos da fase $\alpha$-Fe das amostras nitretadas se encontram levemente deslocados para a região de menor ângulo $2 \theta$ com relação à amostra sem nitretação. Isto indica um parâmetro de rede, referente ao ângulo $\psi=0^{\circ}$ medido, menor do que aquele medido na amostra não nitretada, segundo a Lei de Bragg. A diminuição do parâmetro de rede é proveniente da compactação causada pela formação de nitretos e que geram um aumento de tensão residual na camada nitretada [55]. Esse aumento no nível de tensões residuais na superfície de um componente mecânico melhora a sua vida em fadiga [37,67,68].

A intensidade dos picos $\alpha$-Fe diminuiu com o aumento da espessura da camada de compostos, ao mesmo tempo em que as fases $\varepsilon-\mathrm{Fe}_{2-3}(\mathrm{C}) \mathrm{N}$ e $\gamma^{\prime}-\mathrm{Fe}_{4} \mathrm{~N}$ aumentaram. De acordo com Diehl et al. [55], isso ocorre devido à absorção da radiação pelas porções mais superficiais do material e, portanto, os raios difratados pelas porções mais internas são amenizados. Os picos referentes à fase de nitretos $\gamma^{\prime}-\mathrm{Fe}_{4} \mathrm{~N}$ aumentam com a temperatura de tratamento devido ao aumento de espessura da camada de nitretos $\gamma^{\prime}-\mathrm{Fe}_{4} \mathrm{~N}$ [55]. Por outro lado, a medida que o ângulo $2 \theta$ diminui, também diminui a penetração [69], assim, os picos da fase $\varepsilon-$ $\mathrm{Fe}_{2-3}(\mathrm{C}) \mathrm{N}$ são facilmente identificados com ângulos $2 \theta$ menores. Para ângulos maiores praticamente não se observam os picos da fase $\varepsilon-\mathrm{Fe}_{2-3}(\mathrm{C}) \mathrm{N}$, principalmente em tratamentos na temperatura de $400{ }^{\circ} \mathrm{C}$, pois a camada de compostos é bem fina nestes casos.

Uma explicação para a Figura 4 é a de que com a intensificação da formação de poros, a dureza da camada e compostos é comprometida e por isso o valor de dureza diminui $[55,70]$ para a amostra de $550{ }^{\circ} \mathrm{C}$, por outro lado, a dureza menor para a amostra nitretada a $400{ }^{\circ} \mathrm{C}$ se justifica pelo fato da espessura de camada ser muito pequena. A profundidade modificada nestes casos foi de $308,6 \mu \mathrm{m}$ no tratamento de $550{ }^{\circ} \mathrm{C}$, enquanto que a profundidade modificada no tratamento de $400{ }^{\circ} \mathrm{C}$ foi de apenas $98,5 \mu \mathrm{m}$. A dureza da zona de difusão na região mais próxima da superfície possível de se medir, se mantém constante, dentro de um pequeno desvio, para todas as amostras nitretadas. Isto é devido ao fato de que nestes tratamentos o enriquecimento de nitrogênio é similar [55].

$\mathrm{Na}$ fase inicial de deslizamento, a camada nitretada tem alta rugosidade, e nesta etapa, o coeficiente de atrito tende a diminuir por causa da menor área de contato. A partir do momento em que o coeficiente de atrito se estabilizou, Figura 5, a porção superficial da camada de compostos se quebra em função da pequena espessura de camada, e neste momento é observado um aumento no coeficiente de atrito [55] devido à formação de partículas duras de terceiro corpo no sistema tribológico [61,71].

A observação das trilhas de desgaste mostradas na Figura 6 esclarece alguns pontos sobre o coeficiente de atrito e o volume de desgaste. De modo geral, as amostras nitretadas apresentam uma aparência suave no interior das trilhas, com aspecto de polimento. O perfil transversal das trilhas de desgaste da amostra não nitretada tem baixa resistência ao desgaste em relação a amostra nitretada a plasma na temperatura de $550{ }^{\circ} \mathrm{C}$. A superfície com alta dureza e uma topografia suave após o desgaste inicial resultou no baixo coeficiente de atrito nesta condição [55,57]. Por outro lado, o perfil transversal das trilhas de desgaste das amostras nitretadas nas temperaturas de 400,450 e $500^{\circ} \mathrm{C}$ aumentou em relação à amostra não nitretada, utilizada como referência. Este resultado mostra que a profundidade das trilhas de desgaste destas amostras ultrapassou a camada de compostos. Por este motivo, a camada nitretada afetou a resistência ao desgaste. É possível observar na trilha de desgaste destas amostras a presença de riscos e sulcos, devidos às partículas de material que soltaram da amostra e permaneceram na interface de contato durante o ensaio [55].

\section{CONCLUSÕES}

De acordo com os resultados obtidos, as seguintes conclusões podem ser retiradas:

(1) É possível modificar a superfície do aço DIN 18MnCrSiMo6-4 através da nitretação a plasma, inclusive verificou-se que este aço é compatível com o processo e excelentes resultados foram obtidos no que 
tange a profundidade de camada;

(2) O tratamento de nitretação a plasma contribuiu significativamente para o aumento de dureza superficial, mesmo nas condições em que foi detectada uma fina espessura de camada de compostos;

(3) $\mathrm{O}$ difratograma das amostras nitretadas mostra a presença majoritária da fase de nitretos $\varepsilon-\mathrm{Fe}_{2-3} \mathrm{~N}$ na camada de compostos, e em menor quantidade, a fase de nitretos $\gamma^{\prime}-\mathrm{Fe}_{4} \mathrm{~N}$;

(4) O coeficiente de atrito entre a amostra e a esfera de alumina é mais baixo nas amostras nitretadas no início do ensaio devido a rugosidade da camada ser maior do que a rugosidade da amostra sem nitretação. Após as asperezas serem removidas e o ensaio se estabilizar, o coeficiente de atrito aumentou para as amostras nitretadas na temperatura de 400,450 e $500{ }^{\circ} \mathrm{C}$, e diminuiu para as amostras nitretadas na temperatura de $550{ }^{\circ} \mathrm{C}$;

(5) O perfil transversal das trilhas de desgaste das amostras nitretadas na temperatura 400, 450 e $500{ }^{\circ} \mathrm{C}$ aumentou em relação à amostra não nitretada, utilizada como referência, porém, para as amostras nitretadas na temperatura de $550{ }^{\circ} \mathrm{C}$ o perfil transversal das trilhas de desgaste diminuiu pelo fato da camada de compostos ser mais espessa que o desgaste gerado pela trilha.

\section{AGRADECIMENTOS}

O presente trabalho foi realizado com apoio da Coordenação de Aperfeiçoamento de Pessoal de Nível Superior - Brasil (CAPES) - Código de Financiamento 001 (Programa CAPES BRAGECRIM - Processo $\mathrm{n}^{\circ}$ 88881.142485/2017-01 - Auxílio n 1844/2017), e do Conselho Nacional de Desenvolvimento Científico e Tecnológico - Brasil (CNPq) no âmbito do Processo 311348/2015-7, Produtividade de Pesquisa - PQ 2015.

\section{BIBLIOGRAFIA}

[1] JIN, Y. S. "Development of advanced high strength steels for automotive applications", La Metallurgia Italiana, v. 6, pp. 43-48, 2011.

[2] SSAB TUNNPLAT. Sheet steel handbook: design and fabrication in high strength sheet steel, 3. ed. Sweden, SSAB Tunnplat AB, 1996.

[3] SSAB TUNNPLAT. Sheet steel forming handbook: size shearing and plastic forming, Sweden, SSAB Tunnplat AB, 1998.

[4] SSAB TUNNPLAT. Sheet steel joining handbook: joining of high strength steels, Sweden, SSAB Tunnplat AB, 2004.

[5] NILSSON, T. Welding of AHSS/UHSS steel: a guide for the automotive industry, Sweden, SSAB, 2012. $74 \mathrm{p}$.

[6] ROWE, J. Advanced materials in automotive engineering, Woodhead Publishing Limited, 2012.

[7] DEMERI, M. Y. Advanced high-strength steels: science, technology, and applications, ASM International, 2013.

[8] ZHAO, J., JIANG, Z. "Thermomechanical processing of advanced high strength steels", Progress in Materials Science, v.94, pp. 174-242, 2018.

[9] GALÁN, J., SAMEK, L., VERLEYSEN, P., et al., “Advanced high strength steels for automotive industry", Revista de Metalurgia, v. 48, n. 2, pp. 118-131, 2012.

[10] AKERSTROM, G., RAVENSHORST, H., GUNNARSON, S. “A survey of precipitation hardened pearlitic-ferritic steels in forged automotive components with special emphasis on machinability", Materials \& Design, v.12, n.1, pp. 33-39, 1991.

[11] MATLOCK, D. K., KRAUSS, G., SPEER, J. G. "Microstructures and properties of direct-cooled microalloy forging steels”, Journal of Materials Processing Technology, v. 117, pp. 324-328, 2001.

[12] NUSSBAUM, G. "Strukturbildungsprozesse und struktur/eigenschafts-relationen in mikrolegierten schmiedestählen nach thermomechanischer behandlung mit variierenden abkühlkonzepten", $\mathrm{PhD}$ thesis, RWTH Aachen, 2002.

[13] BRUCHWALD, O., FRACKOWIAK, W., BUCQUET, T., et al., "In-situ-erfassung der werkstoffumwandlung und gefügeausbildung von schmiedebauteilen im abkühlpfad", Journal of Heat Treatment and Materials, v.70, n.3, pp. 150-161, 2015. 
[14] WANG, J., VAN DER WOLK, P. J., VAN DER ZWAAG, S. "On the influence of alloying elements on the bainite reaction in low alloy steels during continuous cooling”, Journal Materials Science, v.35, pp. 4393-4404, 2000.

[15] CABALLERO, F. G., BHADESHIA, H. K. D. H., MAWELLA, K. J. A., et al., "Design of novel high strength bainitic steels: part 1", Materials Science and Technology, v.17, n.5, pp. 512-516, 2001.

[16] CABALlERO, F. G., BHADESHIA, H. K. D. H., MAWELLA, K. J. A., et al., "Design of novel high strength bainitic steels: part 2", Materials Science and Technology, v.17, n.5, pp. 517-522, 2001.

[17] CABALLERO, F. G., CHAO, J., CORNIDE, J., et al., “Toughness deterioration in advanced high strength bainitic steels”, Materials Science and Engineering A, v.525, pp. 87-95, 2009.

[18] RAEDT, H., SPECKENHEUER, U., VOLLRATH, K. "New forged steels: energy-efficient solutions for stronger parts", ATZ autotechnology, v.12, pp. 12-17, 2012.

[19] KEUL, C., WIRTHS, V., BLECK, W. "New bainitic steel for forgings", Archives of Civil and Mechanical Engineering, v.12, pp. 119-125, 2012.

[20] CABALLERO, F. G., GARCIA-MATEO, C., CORNIDE, J., et al., "New advanced ultra high strength bainitic steels: ductility and formability (DUCTAFORM)", Research Fund for Coal and Steel, European Commission, 2013.

[21] SOURMAIL, T., SMANIO, V. ZIEGLER, C., et al., "Novel nanostructured bainitic steel grades to answer the need for high-performance steel components (NANOBAIN)", Research Fund for Coal and Steel Publications, European Commission, 2013.

[22] ROSE, A. J., MOHAMMED, F., SMITH, A. W. F., et al., "Superbainite: laboratory concept to commercial product”, Materials Science Technology, v. 30, n.9, pp. 1094-1098, 2014.

[23] BHADESHIA, H. K. D. H. Bainite in steels: theory and practice, 3. ed. Cambridge, Maney Publisching, 2015. 589 p.

[24] BLECK, W., PRAHL, U., HIRT, G., et al., "Designing new forging steels by ICMPE”, In: Advances in production technology, pp. 85-98, 2015.

[25] CABALlERO, F. G., GARCIA-MATEO, C., MILLER, M. K. "Modern steels at atomic and nanometer scales”, Materials Science and Technology, v.31, n.7, pp. 764-772, 2015.

[26] GLADMAN, T. The physical metallurgy of microalloyed steels, 1. ed. London, Institute of Materials, 1997. $363 \mathrm{p}$.

[27] BHADESHIA, H. K. D. H., HONEYCOMBE, R. W. K. Steel: microstructure and properties, 3. ed. Cambridge, Elsevier LTD, 2006.

[28] DEGARMO, E. P., BLACK, J. T., KOHSER, R. A. Materials and process in manufacturing, 9 ed. Wiley, 2003.

[29] ZAJAC, S., KOMENDA, J., MORRIS, P., et al., "Quantitative structure-property relationship for complex bainitic microstructures. Technical Steel Research report no. EUR 21245EN", European Commission, Luxembourg, 157 p, 2005.

[30] HASLER, S., ROELOFS, H., LEMBKE, M., et al., "New air cooled steels with outstanding impact toughness", In: 3nd International Conference on Steels in Cars and Trucks, Salzburg, June 2011.

[31] CABALLERO, F. G., ROELOFS, H., HASLER, St., et al., "Influence of bainite morphology on impact toughness of continuously cooled cementite free bainitic steels", Materials Science and Technology, v.28, n.1, pp. 95-102, 2012.

[32] MERKEL, C., ENGINEER, J. "Hochfester bainitischer stahl 20MnCrMo7 für umformanwendungen”, Schmiede Journal, pp. 38-41, 2014.

[33] EGGBAUER, G., BUCHMAYR, B. "High-strength bainitic steels for forged products. Berg- und Hüttenmännische Monatshefte”, v.160, n.5, pp. 209-213, 2015.

[34] LEMBKE, M. I., OLSCHEWKI, G., ROELOFS, H., et al., "Nitrieren von hochfesten, bainitischen langprodukten”, Journal of Heat Treatment and Materials, v. 69, n.3, pp. 195-200, 2014.

[35] ROELOFS, H., HASLER, St., URLAU, U., et al., "Continuously cooled bainitic steel HSX®Z12: one decade of experience", In: 4th International Conference on Steels in Cars and Trucks, 2014. 
[36] ABDALLA, A. J., SANTOS, D., VASCONCELOS, G., et al., "Changing in fatigue life of $300 \mathrm{M}$ bainitic steel after laser carburizing and plasma nitriding", In: 12th International Fatigue Congress, MATEC Web of Conferences, 2018.

[37] GENEL, K., DEMIRKOL, M., ÇAPA, M. "Effect of ion nitriding on fatigue behavior of AISI 4140 steel”, Materials Science and Engineering A, v.279, pp. 207-216, 2000.

[38] SUH, C. M., HWANG, J. K., SON, K. S., et al., "Fatigue characteristics of nitrided SACM 645 according to the nitriding condition and notch", Materials Science and Engineering A, v. 392, pp. 31-37, 2005.

[39] ZHANG, P., ZHANG, F. C., YAN, Z. G., et al., "Wear property of low-temperature bainite in the surface layer of a carburized low carbon steel”, Wear, v. 271, pp. 697-704, 2011.

[40] GUESSER, W. L., KODA, F., MARTINEZ, J. A. B., et al., "Austempered ductile iron for gears", SAE Technical Paper Series, n. 2012-36-0305, 2012.

[41] TERRIN, A., DENGO, C., MENEGHETTI. G. "Experimental analysis of contact fatigue damage in case hardened gears for off-highway axles", Engineering Failure Analysis, v.76, pp.10-26, 2017.

[42] RAKHIT, A. K. Heat treatment of gears: a practical guide to engineers, Materials Park, ASM International, 2000.

[43] LESKOVSEK, V., PODGORNIK, B., NOLAN, D. "Modelling of residual stress profiles in plasma nitrided tool steel", Materials Characterization, v.59, pp. 454-461, 2008.

[44] OCHAO, E. A., WISNIVESKY, D., MINEA, T., et al., "Microstructure and properties of the compound layer obtained by pulsed plasma nitriding in steel gears", Surface \& Coatings Technology, v.203, pp. 14571461, 2009.

[45] PODGORNIK, B., LESKOVSEK, V., KOVACIC, M., et al., "Analysis and prediction of residual stresses in nitrided tool steel”, Materials Science Forum, v. 681, pp. 352-357, 2011.

[46] ROLINSKI, E. "Deep nitriding for gear applications and their partial treatments: plasma/ion nitriding meets demanding gear applications by increase load capacity of gears and increased fatigue strength of components as well as less complicated masking treatments" Gears Solutions, 2016. Available in: <http://gearsolutions.com/media/uploads/201605/0516-HS.pdf >. Accessed on: 03 Dec. 2018.

[47] ASTM E3. Standard practice for preparation of metallographic specimens, West Conshohocken, ASTM International, 1995.

[48] GRAZULIS, S., DASKEVIC, A., MERKYS, A., et al., "Crystallography Open Database (COD): an open-access collection of crystal structures and platform for world-wide collaboration", Nucleic Acids Research, v.40(D1), pp. D420-D427, 2012.

[49] KARLSRUHE, F. Inorganic Crystal Structure Database - ICSD, Available in: <https://icsd.fizkarlsruhe.de>. Acessed on: 25 Jan. 2019.

[50] DOEBELIN, N., KLEEBERG, R. "Profex: A graphical user interface for the Rietveld refinement program BGMN", Journal of Applied Crystallography, v.48, pp. 1573-1580, 2015.

[51] ASTM E384. Standard test method for knoop and vickers hardness of materials, West Conshohocken, ASTM International, 2011.

[52] DIN 50 190. Härtetiefe wärmebehandelter Tiele: Ermittlung der Nitrierhärtetiefe. Teil 3. Berlin, DIN Deutsches Institut für Normung, 1979.

[53] CATTEAU, S. D., VAN LANDEGHEM, H. P., TEIXEIRA, J., et al., "Carbon and nitrogen effects on microstructure and kinetics associatedwith bainitic transformation in a low-alloyed steel", Journal of Alloys and Compounds, v. 658, pp. 832-838, 2016.

[54] SUN, Y., BELL, T. "Plasma surface engineering of low alloy steel", Materials Science and Engineering A, v.140, pp. 419-434, 1991.

[55] DIEHL, I. L., DONG, J., ROCHA, A. S. "Propriedades do aço AISI 4140 nitretado a gás", Revista Matéria, v.22, n.4, pp.1-15, 2017.

[56] ALSARAN, A., KARAKAN, M., ÇELIK, A. "The investigation of mechanical properties of ionnitrided AISI 5140 low-alloy steel”, Materials Characterization, v.48, pp. 323-327, 2002.

[57] BOTH, G. B., ROCHA, A. S., SANTOS, G. R., "An investigation on the suitability of different surface treatments applied to a DIN X100CrMoV8-1-1 for cold forming applications", Surface \& Coatings Technology, v. 244, pp. 142-150, 2014. 
[58] LI, Y., HE, Y., XIU, J., et al., "Wear and corrosion properties of AISI 420 martensitic stainless steel treated by active screen plasma nitriding”, Surface \& Coatings Technology, v. 329, pp. 184-192, 2017.

[59] BAI, M., XUE, Q., GUO, H. "Reciprocal sliding wear of SiC particle reinforced Al-Cu aluminium matrix composites against stainless steel, high speed tool steel and ceramics", Wear, v.195, pp. 152-161, 1996.

[60] ÇOMAKLI, O., YETIM, A. F., KARACA, B., et al., "Effect of gas mixture on tribological performance of plasma nitrided grey cast iron under dry and lubricated conditions. Materials Research Express", v.6, n.5, pp.1-11, 2019.

[61] BUDINSKI, K. G. Surface engineering for wear resistance, Upper Saddle River, Prentice-Hall, 1988.

[62] ROCHA, A. S., STROHAECKER, T., HIRSCH, T. "Effect of different surface states before plasma nitriding on properties and machining behavior of M2 high-speed steel", Surface \& Coatings Technology, V.165, pp. 176-185, 2003.

[63] TIER, M., SANTOS, A. V., KUHNEN, C. A., et al., “A study of grain boundary precipitation during plasma nitriding of steel”, In: Proceedings from Surface Modification Technologies XV, Indianapolis, USA, 2001.

[64] KWIETNIEWSKI, C., FONTANA, W., MORAES, C., et al, "Nitrided layer embrittlement due to edge effect on duplex treated AISI M2 high-speed steel”, Surface \& Coatings Technology, v. 179, pp. 27-32, 2004

[65] CZERWINSKI, F. “Thermochemical treatment of metals”, In: Heat Treatment, Conventional and Novel Applications, 2012.

[66] GONG, W., TOMOTA, Y., HARJO, S., et al., "Effect of prior martensite on bainite transformation in nanobainite steel", Acta Materialia, v. 85, pp. 243-249, 2015.

[67] LIMODIN, N., VERREMAN, Y. "Fatigue strength improvement of a 4140 steel by gas nitriding: influence on notch severity", Materials Science \& Engineering A, v.435-436, pp. 460-467, 2006.

[68] SOLEIMANI, S. M. Y., MASHREGUI, A. R., GHASEMI, S. S., et al., "The effect of plasma nitriding on the fatigue behavior of DIN 1.2210 cold work tool steel”, Materials and Design, v. 35, pp. 87-92, 2012.

[69] ROCHA, A. S., STROHAECKER, T., TOMALA, V., et al., "Microstructure and residual stresses of a plasma-nitrided M2 tool steel”, Surface \& Coatings Technology, v.115, pp. 24-31, 1999.

[70] SKONIESKI, A. F. O., SANTOS, G. R., et al., "Metallurgical response of an AISI 4140 steel to different plasma nitriding gas mixtures", Materials Research, v. 16, n.4, pp. 884-890, 2013.

[71] LEMM, J. D., WARMUTH, A. R., PEARSON, S. R., et al., "The influence of surface hardness on the fretting wear of steel pairs - Its role in debris retention in the contact", Tribology International, v. 81, pp. 258-266, 2015.

\section{ORCID}

Rafael Luciano Dalcin Leonardo Fonseca Oliveira Alexandre da Silva Rocha Célia de Fraga Malfatti Gelsa Edith Navarro Hidalgo Jérémy Epp Hans-Werner Zoch https://orcid.org/0000-0002-5724-7114 https://orcid.org/0000-0003-4670-9157 https://orcid.org/0000-0002-4086-5389 https://orcid.org/0000-0002-0819-479X https://orcid.org/0000-0003-4851-7415 https://orcid.org/0000-0003-4140-926X https://orcid.org/0000-0002-4347-4746 